\title{
Effects of short-term capture on the physiology of white sharks Carcharodon carcharias: amino acids and fatty acids
}

\author{
Austin J. Gallagher ${ }^{1, *}$, Lauren Meyer ${ }^{2}$, Heidi R. Pethybridge ${ }^{3}$, Charlie Huveneers ${ }^{2}$, \\ Paul A. Butcher ${ }^{4}$ \\ ${ }^{1}$ Beneath the Waves, PO Box 126, Herndon, VA 20172, USA \\ ${ }^{2}$ Southern Shark Ecology Group, College of Science and Engineering, Flinders University, Bedford Park, SA 5042, Australia \\ ${ }^{3}$ CSIRO Oceans and Atmosphere, Hobart, TAS 7004, Australia \\ ${ }^{4}$ NSW Department of Primary Industries, National Marine Science Centre, Coffs Harbour, NSW 2450, Australia
}

\begin{abstract}
Empirical evaluations of how overexploited marine fishes respond to capture stress (physiologically and behaviourally) have become increasingly important for informed fisheries management. These types of studies are, however, lacking for many protected species. Here, we conducted a novel study on the physiology of juvenile white sharks Carcharodon carcharias (139-325 cm fork length), a globally protected and ecologically important predator, in response to a standard fishery interaction using shark-management-alert-in-real-time (SMART) drumlines as part of a bather protection program. Specifically, we assessed the influence of short-term capture duration (average: $30 \mathrm{~min}$; range: 10-75 $\mathrm{min}$ ) and other biological (size) and environmental (temperature) variables on blood plasma amino acids and fatty acids, which play essential roles as energy substrates as well as in maintaining physiological functions. None of the assessed amino acids or fatty acids were affected by capture duration, but some were influenced by shark size and water temperature. Our results support the notion that white shark physiology is robust to capture at short capture durations, which has important implications for the fate of released individuals.
\end{abstract}

KEY WORDS: Amino acid - Capture - Fatty acid · Fishing - Shark - Stress · Biochemistry · Ecophysiology $\cdot$ White shark

\section{INTRODUCTION}

Understanding the effects of capture on elasmobranchs is important for accurately evaluating the impacts of fisheries interactions, especially for oceanic species which are vulnerable to bycatch and are at high extinction risk (Gallagher et al. 2012, Dulvy et al. 2014). Previous work has demonstrated a range of species- and gear-specific responses to fisheries capture, ranging from physiological disruption to post-release mortality, underscoring the complexity of this issue (e.g. Mandelman \& Skomal 2009, Marshall et al. 2012, Butcher et al. 2015, Dapp et al.

\footnotetext{
${ }^{*}$ Corresponding author: austin@beneaththewaves.org
}

2016b). In recent years, studies examining the physiological responses and post-capture behaviour of elasmobranchs, particularly sharks, have expanded from a common set of commercially relevant species subjected to longline fishing (Dapp et al. 2016a) to work on coastal species under recreational and fishery-independent contexts (Danylchuk et al. 2014, Gallagher et al. 2017a, Whitney et al. 2017, Jerome et al. 2018), coral reef-dwelling species (Dapp et al. 2017), polar settings (Barkley et al. 2017), and even the deep sea (Talwar et al. 2017). In addition to advancing our understanding of the comparative physiology of elasmobranchs, this research provides

() The authors 2019. Open Access under Creative Commons by Attribution Licence. Use, distribution and reproduction are unrestricted. Authors and original publication must be credited. 
managers, fishers, and the general public with relevant information to address targeted conservation problems, should they occur.

Despite growth in this field, certain species of large elasmobranchs which are either critically threatened, globally protected, or tightly regulated remain overlooked. Highly threatened species such as whale sharks Rhincodon typus and manta rays Mobula spp., which are listed on Appendix II of the Convention on International Trade in Endangered Species of Wild Fauna and Flora (CITES) and are incidentally captured in nets, have not been evaluated for their capture stress. The smalltooth sawfish Pristis pectinata, which was the world's first elasmobranch species to be listed on the US Endangered Species list in 2003, was only recently evaluated for its physiological response to capture (Prohaska et al. 2018), while a recent study assessed the fate of pygmy devilray, Mobula kuhlii cf. eregoodootenkee (listed on CITES Appendix II in 2017) caught in the NSW (Australia) shark control program (Broadhurst \& Cullis 2019). These data can help the conservation community understand the relative contribution that captureinduced stress and ensuing post-release mortality could have on threatened species when caught and released. Such information is valuable when undertaking ecosystem-based assessments of fisheries catching threatened species as bycatch and during conservation assessments (e.g. IUCN Red List). For example, we now know that capture-induced mortality likely played a role in the population declines of threatened hammerhead shark species Sphyrna spp. (Gallagher et al. 2014a,b, Gulak et al. 2015). This information can also help researchers understand how their procedures may affect threatened species.

White sharks Carcharodon carcharias are the largest species of predatory shark. They are globally distributed, with hotspots in South Africa, Australia, New Zealand, Mexico, and the United States (Jorgensen et al. 2010, Kock et al. 2013, Skomal et al. 2017, Nazimi et al. 2018). They generate significant public attention and study from researchers (Huveneers et al. 2018), evidenced by a study bias in many areas (Hammerschlag et al. 2011, Ducatez 2019), and they are a primary target of a lucrative global tourism industry (Huveneers et al. 2017, Gallagher \& Huveneers 2018). White sharks are protected throughout all of these hotspots and are listed on Appendix II of CITES. However, historical concerns about beach and bather safety in locations such as Australia and South Africa have prompted controversial shark exclusion and removal techniques resulting in decades of white shark captures (Cliff et al. 1989, Cliff \& Dud- ley 1992, Reid et al. 2011). In addition, incidental catches of white sharks still occur in commercial and recreational fisheries (Malcolm et al. 2001, Lowe et al. 2012, Dicken \& Booth 2013, Lyons et al. 2013, Curtis et al. 2014). Thus, despite receiving near-global protection from virtually all types of fisheries, the number of white sharks being captured each year may not be trivial - inclusive of fishery-independent research operations. To date, only a single study has evaluated white shark response to capture, using basic blood bio-chemical markers. This study concluded that short-term capture did not affect most of the standard variables indicative of a stress response (Tate et al. 2019). However, it remains unknown whether this conclusion holds true across a broader suite of physiological variables that may signal the transport of energy to tissues. This would provide greater resolution to the paucity of information on white shark responses to fisheries interactions.

In recent years, researchers have begun evaluating novel parameters as potential biomarkers of stress in elasmobranch fishes (e.g. Van Rijn \& Reina 2010, Guida et al. 2016a). Biochemical markers such as amino acids and fatty acids (FAs) could give novel insights into the individual-level mechanisms that govern how organisms respond to stress and environmental variation. Under stress, organisms can have additional metabolite requirements due to higher energy demands or secondary stress responses, such as the synthesis of stress-related proteins or hormones. In a number of fish species, repeated acute handling has been shown to affect both the mobilization of amino acids (Aragão et al. 2008, Costas et al. 2011) and plasma free FAs (Mazeaud et al. 1977). Other environmental stressors, such as temperature (e.g. cold acclimation), have also been reported to alter plasma lipid and amino acid concentrations (Hsieh et al. 2003, Ma et al. 2015). Elasmobranch fishes have an unusual lipid metabolism whereby FAs are largely stored in their large livers, which is where most $\beta$-oxidation occurs (Ballantyne 1997). Therefore, products such as ketone bodies may be more heavily relied upon as a source of energy than FAs (Gallagher et al. 2017b). In dogfish sharks (Squalidae), plasma concentrations of amino acids and non-esterified FAs have been shown to change in response to feeding and fasting (Wood et al. 2010). Certain FAs (i.e. saturated fatty acids [SFAs] and monounsaturated fatty acids [MUFAs]) are more metabolically active than others (i.e. polyunsaturated fatty acids [PUFAs]; Tocher 2003), and as such we would anticipate a change in the FA profiles associated with capture stress. A few studies 
have explored changes in specific FAs with teleost migrations (Bell et al. 1986, Osako et al. 2006), temperature (Gladyshev et al. 2018), and energy expenditure (exercise in $\mathrm{Li}$ et al. 2016; swimming velocity in Gladyshev et al. 2018). Thus, it appears that amino acids and FAs could serve as informative indicators of the secondary stress response in sharks. While the capture of sharks and rays has been shown to influence blood glucose, lactate, and $\mathrm{pH}$ (Moyes et al. 2006, Brooks et al. 2012, Heard et al. 2014, Jerome et al. 2018), no studies have examined if capture influences amino acid and FA metabolism in sharks despite the growing popularity of these biomarkers in chondrichthyan ecology (Munroe et al. 2018, Meyer et al. 2019), leaving a gap in our understanding of how these analytes may be influenced by external stressors.

Here, we conducted an empirical evaluation of the physiological response of white sharks to capture on shark management alert in real-time (SMART) drumlines, focusing on individuals opportunistically sampled from a shark management program off New South Wales (NSW), Australia. Our study had 2 primary objectives: (1) evaluate and describe an amino acid and FA profile from white shark blood plasma; and (2) evaluate whether these parameters were affected by short-term fisheries capture, and by a set of additional biotic and abiotic variables. We discuss our findings in relation to shark metabolic strategies, provide comparisons to results obtained from other species of large sharks, and make recommendations as to how fisheries interactions might affect this threatened species.

\section{MATERIALS AND METHODS}

\subsection{Fishing gear and animal capture}

White sharks were captured, sampled, and released over a 13 mo period between July 2016 and August 2017 onboard research vessels off the midnorth and northern NSW coast, Australia. Sampling was opportunistic, capitalizing on the capture of white sharks on SMART drumlines as a part of a broader research tagging and tracking program associated with a jurisdictional Shark Management Strategy in NSW.

SMART drumlines were configured following Tate et al. (2019). Various semi-barbed and barbed hooks were used as part of other ongoing research with SMART drumlines, but all were baited with 0.75-1 kg of sea mullet Mugil cephalus. A $2.0 \mathrm{~m}$
(2 mm $\varnothing$ ) monofilament 'trigger line' was attached between the elasticized cord and the SMART buoy. When an animal bit the hook, the trigger line separated the magnet in the SMART buoy and a signal was transmitted via satellite, alerting researchers via SMS, telephone call, and email. On each fishing day, SMART drumlines were deployed $\sim 500 \mathrm{~m}$ from shore in $\sim 4-15 \mathrm{~m}$ water depth during daylight hours. Once an alert was received, thus beginning the capture period used to approximate 'hook time', the research vessel would travel to the SMART drumline and monitor the gear and sharks' activity. If multiple alerts came through, the first shark to trigger the system was attended to first. Each shark was approached once it maintained a normal upright swimming position without fighting the 'drumline' buoy. Once the SMART drumline was retrieved, the trace was attached to a longer rope so the shark could be secured to the side of the vessel with that and an additional (1) cross-pectoral fin and (2) tail rope which contained a PVC sleeve to minimize the chance of damaging the shark. Shark sex and size (fork length [FL], to the nearest $\mathrm{cm}$ ) was recorded.

\subsection{Sample extraction and laboratory analyses}

Whole blood ( 5-8 ml) was sampled via a nonlethal caudal venipuncture biopsy using a $90 \mathrm{~mm}, 18$ gauge needle and a $10 \mathrm{ml}$ syringe according to Butcher et al. (2015), within 1 min of being brought alongside the boat. The timing of the total capture event ended once the blood sample was taken, thereby creating a continuous variable for testing the effects of capture on blood physiology. The blood was transferred to an $8 \mathrm{ml}$ plasma separator tube containing lithium heparin (BD Vacutainer) and was stored on ice temporarily before being centrifuged at $5000 \mathrm{rpm}$ for $4 \mathrm{~min}$. Plasma samples were then separated into three $2 \mathrm{ml}$ vials and immediately frozen at $-18^{\circ} \mathrm{C}$ in the field before being transferred to a $-80^{\circ} \mathrm{C}$ freezer in the laboratory.

Amino acids profiles were quantified by IDEXX Laboratories (Brisbane, Australia) within $7 \mathrm{~d}$ to prevent blood chemistry alterations (Barton et al. 2002). Amino acids were divided into 4 groups: (1) ketone body $\beta$-hydroxybutyrate; (2) branched-chain essential amino acids: isoleucine, leucine, and valine; (3) other essential amino acids: histidine, lysine, phenylalanine, and threonine; and (4) non-essential amino acids: alanine, aspartic acid, glutamine, glycine, proline, serine, and tyrosine. 
Lipids and FAs were extracted from plasma according to the methods detailed by Bligh \& Dyer (1959) and Meyer et al. (2017), respectively. This included using various solvent solutions to separate lipids from proteins and carbohydrates (of dichloromethane, methanolde-ionized water) and then to separate individual FAs from the glycerol backbones of polar and non-polar lipids (with methanol, hexane, and hydrochloric acid). Total lipid content was gravimetrically measured and individual FAs were identified and quantified through gas chromatographic analysis using an Agilent Technologies 6890N gas chromatograph fitted with an HP-5 cross-linked methyl silicone fused silica capillary column $(50 \times$ $0.32 \mathrm{~mm}$ i.d.), a flame ionization detector (FID), a splitless injector, and an Agilent Technologies 7683 Series auto-sampler. Selected samples were further analyzed by GC-MS on a Finnigan Thermoquest system fitted with an on-column injector for peak verification. FA results are expressed as a proportion of the total identified compounds. Only FAs over $0.5 \%$ (25 out of 61) were included in statistical analyses.

\subsection{Data collected and statistical analysis}

We tested the influence of capture duration, shark sex and size, and water temperature on white shark plasma FA and amino acid profiles, using multivariate statistical analyses undertaken in PRIMER7 +PERMANOVA (Clarke \& Gorley 2015). Permutational multivariate analysis of variance (PERMANOVA) with Monte Carlo simulations, denoted as $\mathrm{p}(\mathrm{MC})$, were run on Bray-Curtis similarity matrices calculated from the square-root-transformed profile data to determine if factors significantly influenced the overall FA and amino acid profiles. Continuous factors (capture duration, shark length, and water temperature) were classified as covariates, and sex as a random categorical variable in the PERMANOVA model containing all 4 factors. Significance was declared at $\mathrm{p}(\mathrm{MC})<0.05$. A canonical analysis of principal coordinates (CAP) (Anderson \& ter Braak 2003) of the Bray-Curtis similarity matrix was also run to visualize trends in FA and amino acid profiles across capture time and FL.

We then assessed if individual plasma amino acids and FAs were influenced by capture duration, while accounting for biological (shark sex and size) and environmental (water temperature) variables using a generalized linear model (GLM). We determined the most appropriate statistical family and error distribution (family = gamma; link = log) by examining the distribution of the response variable and visually inspecting the residuals for the saturated models. Statistical significance was declared at $\mathrm{p}<0.05$; these analyses were performed in RStudio (R Core Team 2016).

\section{RESULTS}

A total of 52 white sharks (47 individuals, 5 recaptures) were sampled for blood during the study period (Table 1). This pool of sharks included 33 females (mean \pm SD: $229 \pm 45 \mathrm{~cm} \mathrm{FL)} \mathrm{and} 19$ males (228 \pm $45 \mathrm{~cm} \mathrm{FL})$. Hook times ranged between 10 and $75 \mathrm{~min}$ $(29.8 \pm 15.2 \mathrm{~min} ;$ Table 1$)$. The elapsed duration between the time a shark was secured at the boat to completion of blood collection was $3.0 \pm 2.0 \mathrm{~min}$ (1-13 min). One male and 4 females were recaptured and resampled, with time between capture events being 48, 60, 272, 273, and $244 \mathrm{~d}$, respectively (Table 1).

\subsection{Amino acids}

A summary of the means and ranges for the 15 amino acids assessed here are presented in Table 2. White shark plasma amino acid profiles were not affected by capture duration $(\mathrm{p}[\mathrm{MC}]=0.593$; Fig. $1 \mathrm{~A})$, sex $(\mathrm{p}[\mathrm{MC}]=0.306)$ or water temperature $(\mathrm{p}[\mathrm{MC}]=0.788)$, but were influenced by shark length $(\mathrm{p}[\mathrm{MC}]=0.043 ;$ Fig. 1B). Modelling of individual amino acids revealed none were affected by capture duration (Table 3), nor were any of the essential amino acids (branched-chain or other) affected by the biological or environmental factors. The only factor that affected several amino acids was shark length (see Fig. S1 in the Supplement at www.intres.com/articles/suppl/n040p297_supp.pdf). Specifically, ketone bodies were negatively influenced by shark length $\left(t_{1,52}=-2.26, \mathrm{p}<0.05\right.$; Table 3, Fig. S1). Non-essential amino acids were also influenced by shark length with alanine positively influenced $\left(t_{1,52}=2.71, \mathrm{p}<0.01\right.$; Table 3, Fig. S1), whereas aspartic acid $\left(t_{1,52}=2.06, \mathrm{p}<0.05\right.$; Table 3 , Fig. S1) and glycine $\left(t_{1,52}=-2.68, \mathrm{p}<0.05\right.$; Table 3 , Fig. S1) were negatively affected. Tyrosine was the only amino acid influenced (positively) by water temperature $\left(t_{1,52}=2.31, \mathrm{p}<0.05\right.$; Table 3, Fig. S1).

\subsection{Fatty acids}

White shark plasma FA profiles were dominated by PUFAs $(40.79 \pm 1.45)$, contributing 1.5 and 1.6 times 
Table 1. Summary data of all captured white sharks caught on shark-management-alert-in-real-time (SMART) drumlines from the present study. FA: fatty acid

\begin{tabular}{|c|c|c|c|c|c|c|}
\hline $\begin{array}{l}\text { Date } \\
\text { (dd/mm/yyyy) }\end{array}$ & Location & Sex & $\begin{array}{c}\text { Fork length } \\
\text { (cm) }\end{array}$ & $\begin{array}{l}\text { Water temp. } \\
\left({ }^{\circ} \mathrm{C}\right)\end{array}$ & $\begin{array}{l}\text { Hook time } \\
\text { (min) }\end{array}$ & FA analysis \\
\hline $28 / 11 / 2016$ & Ballina & $\mathrm{F}$ & 252 & 19.4 & 36 & Yes \\
\hline $21 / 07 / 2016$ & Tuncurry & $\mathrm{F}$ & 192 & 19 & 23 & Yes \\
\hline $21 / 07 / 2016$ & Tuncurry & M & 235 & 19.2 & 13 & Yes \\
\hline $21 / 07 / 2016$ & Tuncurry & M & 179 & 19.4 & 19 & Yes \\
\hline $22 / 07 / 2016$ & Tuncurry & $\mathrm{F}$ & 255 & 19.4 & 32 & Yes \\
\hline $27 / 07 / 2016$ & Crowdy Head & $\mathrm{F}$ & 201 & 19 & 24 & Yes \\
\hline 01/08/2016 & Coffs Harbour & M & 259 & 19.2 & 26 & Yes \\
\hline 01/08/2016 & Coffs Harbour & $\mathrm{F}$ & 190 & 19.4 & 21 & No \\
\hline 02/08/2016 & Coffs Harbour & M & 234 & 19.3 & 39 & Yes \\
\hline 09/08/2016 & Ballina & $\mathrm{F}$ & 193 & 20.6 & 30 & No \\
\hline 09/08/2016 & Ballina & $\mathrm{F}$ & 277 & 20.8 & 23 & Yes \\
\hline 09/08/2016 & Ballina & $\mathrm{F}$ & 223 & 20.9 & 27 & Yes \\
\hline 06/09/2016 & Tuncurry & $\mathrm{F}$ & 197 & 18.7 & 23 & No \\
\hline 07/09/2016 & Tuncurry & $\mathrm{F}$ & 230 & 18.5 & 25 & Yes \\
\hline 07/09/2016 & Tuncurry & M & 192 & 18.8 & 19 & Yes \\
\hline 07/09/2016 & Tuncurry & M & 226 & 18.9 & 27 & Yes \\
\hline 07/09/2016 & Tuncurry & $\mathrm{F}$ & 139 & 18.9 & 16 & Yes \\
\hline 08/09/2016 & Tuncurry & M & 174 & 19.2 & 28 & Yes \\
\hline $27 / 09 / 2016$ & Ballina & M & 252 & 19.3 & 24 & Yes \\
\hline $28 / 09 / 2016$ & Ballina & M & 160 & 19.3 & 31 & Yes \\
\hline $01 / 10 / 2016$ & Ballina & M & 187 & 18.9 & 21 & Yes \\
\hline $02 / 10 / 2016$ & Ballina & $\mathrm{M}$ & 240 & 19.1 & 18 & Yes \\
\hline 02/10/2016 & Ballina & M & 268 & 19 & 75 & Yes \\
\hline $02 / 10 / 2016$ & Ballina & M & 218 & 19.2 & 29 & Yes \\
\hline $04 / 10 / 2016$ & Ballina & $\mathrm{F}$ & 300 & 18.4 & 38 & Yes \\
\hline 04/10/2016 & Ballina & $\mathrm{F}$ & 202 & 18.3 & 10 & Yes \\
\hline 06/10/2016 & Ballina & M & 257 & 19.4 & 25 & Yes \\
\hline 08/10/2016 & Ballina & $\mathrm{F}$ & 222 & 19.1 & 27 & Yes \\
\hline 08/10/2016 & Ballina & $\mathrm{F}$ & 192 & 19.8 & 28 & No \\
\hline 08/10/2016 & Ballina & $\mathrm{F}$ & 187 & 19.6 & 22 & Yes \\
\hline $15 / 10 / 2016$ & Ballina & $\mathrm{F}$ & 276 & 22.3 & 22 & Yes \\
\hline $18 / 10 / 2016$ & Ballina & $\mathrm{F}$ & 206 & 21.2 & 27 & Yes \\
\hline $22 / 11 / 2016$ & Ballina & $\mathrm{F}$ & 255 & 20.7 & 53 & Yes \\
\hline $29 / 11 / 2016$ & Ballina & $\mathrm{F}$ & 300 & 19.5 & 23 & Yes \\
\hline $29 / 11 / 2016$ & Ballina & $\mathrm{F}$ & 325 & 19.7 & 39 & Yes \\
\hline 03/12/2016 & Tuncurry & $\mathrm{F}$ & 224 & 22.3 & 25 & Yes \\
\hline 08/12/2016 & Ballina & M & 187 & 20 & 54 & No \\
\hline $10 / 12 / 2016$ & Ballina & M & 245 & 20.4 & 20 & Yes \\
\hline $10 / 12 / 2016$ & Evans Head & $\mathrm{F}$ & 212 & 21.3 & 28 & Yes \\
\hline $10 / 12 / 2016$ & Evans Head & $\mathrm{F}$ & 232 & 21.3 & 71 & Yes \\
\hline $30 / 05 / 2017$ & Coffs Harbour & $\mathrm{M}$ & 240 & 20.8 & 20 & No \\
\hline $06 / 06 / 2017$ & Tuncurry & $\mathrm{F}$ & 220 & 19.9 & 28 & No \\
\hline 06/06/2017 & Tuncurry & $\mathrm{F}$ & 290 & 20 & 71 & No \\
\hline 06/06/2017 & Tuncurry & $\mathrm{F}$ & 251 & 20.1 & 20 & No \\
\hline $06 / 06 / 2017$ & Tuncurry & $\mathrm{F}$ & 223 & 19.6 & 44 & No \\
\hline 06/06/2017 & Tuncurry & $\mathrm{F}$ & 245 & 20.1 & 13 & No \\
\hline $07 / 06 / 2017$ & Tuncurry & M & 221 & 19.9 & 16 & No \\
\hline
\end{tabular}

the SFAs $(27.18 \pm 0.64)$ and MUFAs $(25.12 \pm 1.48)$ to the overall profiles, respectively. PUFA 22:6ஸ3 (17.47 \pm 0.91 ) was the greatest single FA contributor, followed by SFA 16:0 (14.85 \pm 0.38$)$ and MUFA 18:1 109

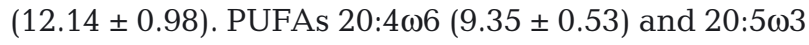
$(6.19 \pm 0.31)$ and SFA 18:0 (7.99 \pm 0.35$)$ were also substantial contributors, with values $>5 \%$ (Table 4 ).
The plasma FA profiles were driven by biological factors, primarily shark length $(\mathrm{p}[\mathrm{MC}]=0.001$; Fig. 2B) and sex $(\mathrm{p}[\mathrm{MC}]=0.01)$. Capture duration and water temperature did not measurably affect the FA profiles $(\mathrm{p}[\mathrm{MC}]=0.989$ and 0.127 , respectively; Fig. 2). Modelling of individual FAs revealed that none were affected by capture duration, while sev- 
Table 2. Plasma amino acid profiles $\left(\mathrm{mmol} \mathrm{l}^{-1}\right)$ from white sharks caught on SMART drumlines in the present study

\begin{tabular}{|lcc|}
\hline Amino acid & Mean $\pm \mathrm{SE}$ & Range \\
\hline$\beta$-hydroxybutyrate & $0.32 \pm 0.03$ & $0.1-1$ \\
Branched-chain essential & & \\
Isoleucine & $177.8 \pm 8.47$ & $99-368$ \\
Leucine & $301.8 \pm 13.75$ & $158-596$ \\
Valine & $375.9 \pm 17.16$ & $202-809$ \\
Other essential & & \\
Histidine & $65.34 \pm 2.90$ & $33-139$ \\
Lysine & $407.6 \pm 26.18$ & $182-1169$ \\
Phenylanaline & $83.87 \pm 3.81$ & $41-164$ \\
Threonine & $208.1 \pm 12.54$ & $59-431$ \\
Non-essential & & \\
Alanine & $1004.0 \pm 59.45$ & $442-2174$ \\
Aspartic acid & $40.89 \pm 5.17$ & $4-165$ \\
Glutamine & $260.90 \pm 12.65$ & $119-541$ \\
Glycine & $111.1 \pm 10.15$ & $36-435$ \\
Proline & $111.8 \pm 8.12$ & $28-262$ \\
Serine & $361.0 \pm 19.54$ & $189-834$ \\
Tyrosine & $114.1 \pm 4.31$ & $63-211$ \\
\hline
\end{tabular}

eral were influenced by abiotic and biotic variables (Table 5, Fig. S2).

\section{DISCUSSION}

Fish exhibit some of the most pronounced stress responses of all vertebrates (Barton et al. 2002). The extent or magnitude of the acute stress response in fishes can provide essential information on their physiological sensitivity to fisheries interactions while providing insights into the ecological and evolutionary drivers of animal performance and metabolism (Calow \& Forbes 1998, Gallagher et al. 2017a). Our results support the notion that white shark physiology is minimally affected by short-term (i.e. $<1.5 \mathrm{~h}$ ) capture durations on SMART drumlines. This conclusion is corroborated by recent work evaluating blood biochemistry and gas tensions under similar contexts (Tate et al. 2019).

Interestingly, none of the amino acids or FAs were affected by capture duration in the present study (Tables 3 \& 5). This result is in contrast to work on teleost species, which have demonstrated strong effects of stress on amino acid and FA metabolism (e.g. Aragão et al. 2008, Li et al. 2016, Gladyshev et al. 2018). Exhaustive exercise has been shown to increase and mobilize amino acids into the plasma of various freshwater teleost species, which is thought to be related to the release of glucocorticoid hormones in the primary stress response (Milligan 1997, Vijayan et al. 1997). Similarly, stress-linked hor- mones have been shown to mobilize FAs which can serve as energy substrates in fish (Wendelaar-Bonga 1997). Branched-chain essential amino acids, which were evaluated in the present study, are important energy substrates in fish, particularly under anaerobic conditions (Li et al. 2009). Ketones are thought to serve as a primary source of metabolic fuel in sharks, largely due to fact that $\beta$-oxidation of FAs is confined to the liver (due to the lack of FA bind proteins in the plasma), whereby FAs are oxidized into ketone bodies and transported for energy (Metcalf \& Gemmell 2005). However, it is unknown whether amino acids and FAs in elasmobranchs (sharks and rays) are influenced by capture and stress similarly to teleosts. Recent work evaluating the effects of air exposure on Atlantic stingrays Hypanus sabinus did not find an effect on ketone bodies, whereas other metrics such
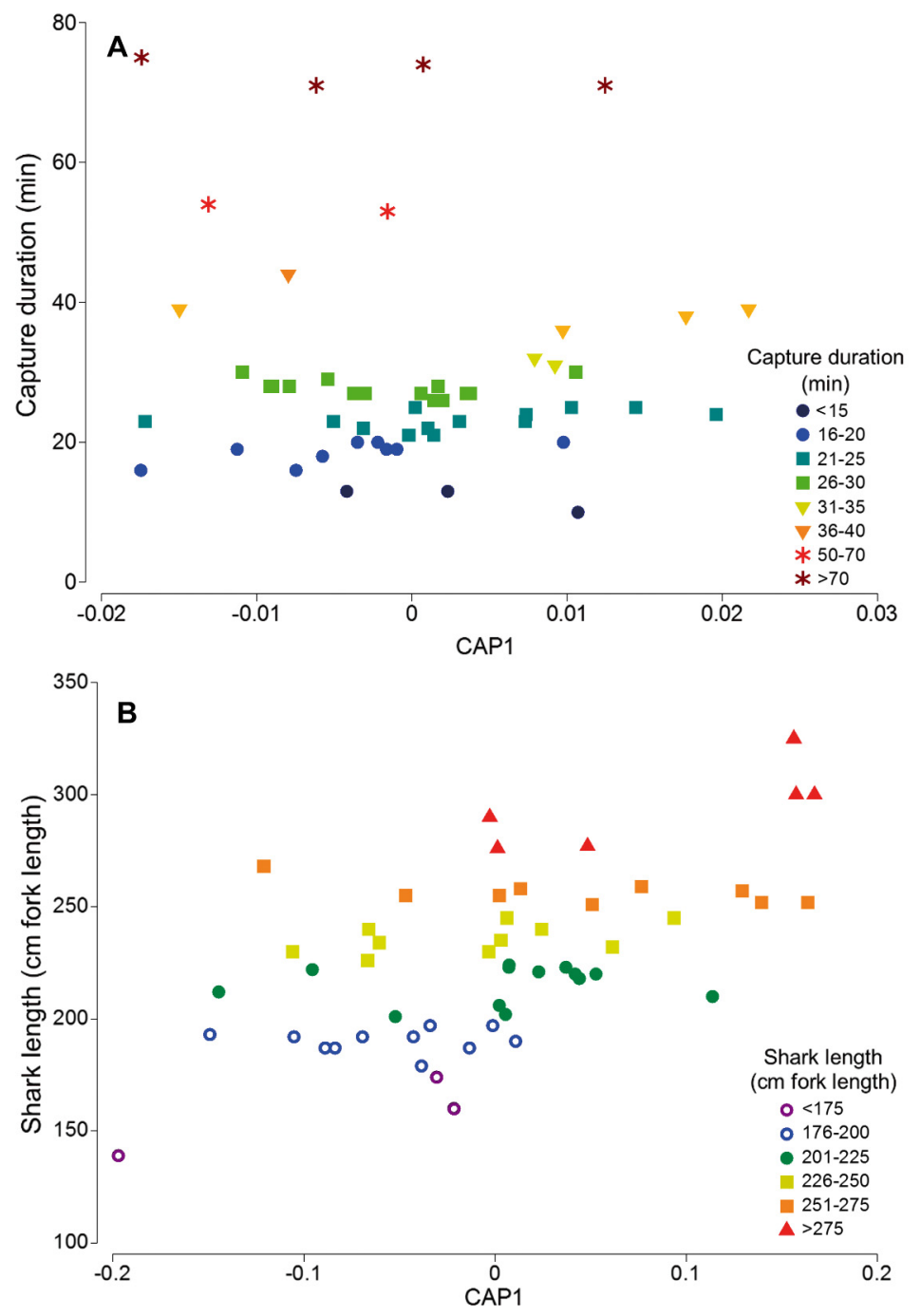

Fig. 1. Canonical analysis of principal coordinates (CAP) of white shark blood plasma amino acid profiles against (A) capture duration and (B) shark length classed as covariates 
Table 3. Generalized linear model results testing the effects of biological and operational variables on each of the 15 plasma amino acids from white sharks caught on SMART drumlines in the present study. Significant p-values $(<0.05)$ in bold. Est: estimate; Inter: intercept; Time: hook time; FL: fork length; Temp: temperature

\begin{tabular}{|c|c|c|c|c|c|}
\hline Response & Parameter & - Est & $\mathrm{SE}$ & $t$ & $\mathrm{p}$ \\
\hline \multirow[t]{5}{*}{$\beta$-hydroxybutyrate } & Inter & 1.06 & 1.94 & 0.55 & 0.587 \\
\hline & Time & 0.00 & 0.01 & 0.01 & 0.994 \\
\hline & Sex & -0.07 & 0.18 & -0.38 & 0.704 \\
\hline & FL & -0.01 & 0.00 & -2.59 & 0.013 \\
\hline & Temp & -0.04 & 0.09 & -0.41 & 0.684 \\
\hline \multirow[t]{5}{*}{ Isoleucine } & Inter & 5.15 & 1.23 & 4.19 & 0.000 \\
\hline & Time & 0.00 & 0.00 & -0.50 & 0.619 \\
\hline & Sex & 0.01 & 0.11 & 0.11 & 0.913 \\
\hline & FL & 0.00 & 0.00 & 0.65 & 0.519 \\
\hline & Temp & -0.01 & 0.60 & -0.13 & 0.890 \\
\hline \multirow[t]{5}{*}{ Leucine } & Inter & 6.10 & 1.19 & 5.14 & 0.000 \\
\hline & Time & 0.00 & 0.00 & -0.50 & 0.617 \\
\hline & Sex & -0.01 & 0.11 & -0.08 & 0.935 \\
\hline & FL & 0.00 & 0.00 & 0.54 & 0.595 \\
\hline & Temp & -0.02 & 0.06 & -0.43 & 0.668 \\
\hline \multirow[t]{5}{*}{ Valine } & Inter & 6.05 & 1.18 & 5.11 & 0.000 \\
\hline & Time & 0.00 & 0.00 & -0.47 & 0.638 \\
\hline & Sex & -0.01 & 0.11 & -0.08 & 0.940 \\
\hline & FL & 0.00 & 0.00 & 1.32 & 0.193 \\
\hline & Temp & -0.02 & 0.06 & -0.43 & 0.669 \\
\hline \multirow[t]{5}{*}{ Histidine } & Inter & 3.07 & 1.14 & 2.68 & 0.104 \\
\hline & Time & 0.00 & 0.00 & 0.55 & 0.589 \\
\hline & Sex & 0.02 & 0.11 & 0.16 & 0.878 \\
\hline & FL & 0.00 & 0.00 & 0.52 & 0.609 \\
\hline & Temp & 0.04 & 0.06 & 0.80 & 0.431 \\
\hline \multirow[t]{5}{*}{ Lysine } & Inter & 4.73 & 1.69 & 2.87 & 0.006 \\
\hline & Time & 0.01 & 0.01 & 0.12 & 0.907 \\
\hline & Sex & -0.02 & 0.15 & -0.13 & 0.897 \\
\hline & FL & 0.00 & 0.00 & 0.24 & 0.814 \\
\hline & Temp & 0.06 & 0.08 & 0.75 & 0.458 \\
\hline \multirow[t]{5}{*}{ Phenylanaline } & Inter & 3.89 & 1.12 & 3.46 & 0.001 \\
\hline & Time & 0.01 & 0.00 & 1.46 & 0.152 \\
\hline & Sex & -0.12 & 0.10 & -1.13 & 0.266 \\
\hline & FL & 0.00 & 0.00 & 0.88 & 0.266 \\
\hline & Temp & 0.01 & 0.05 & 0.26 & 0.797 \\
\hline \multirow[t]{5}{*}{ Threonine } & Inter & 4.91 & 1.50 & 3.28 & 0.002 \\
\hline & Time & 0.00 & 0.00 & -0.46 & 0.645 \\
\hline & Sex & 0.10 & 0.14 & 0.78 & 0.459 \\
\hline & FL & 0.00 & 0.00 & 0.82 & 0.415 \\
\hline & Temp & 0.00 & 0.07 & 0.01 & 0.989 \\
\hline \multirow[t]{5}{*}{ Alanine } & Inter & 8.39 & 1.31 & 6.38 & 0.000 \\
\hline & Time & 0.00 & 0.00 & -0.30 & 0.765 \\
\hline & Sex & -0.10 & 0.12 & -0.79 & 0.434 \\
\hline & FL & 0.00 & 0.00 & 2.71 & 0.010 \\
\hline & Temp & -0.12 & 0.06 & -1.82 & 0.076 \\
\hline \multirow[t]{5}{*}{ Aspartic acid } & Inter & 9.98 & 3.13 & 3.19 & 0.003 \\
\hline & Time & 0.01 & 0.01 & 0.60 & 0.554 \\
\hline & Sex & -0.18 & 0.29 & -0.61 & 0.543 \\
\hline & FL & -0.01 & 0.00 & -1.99 & 0.054 \\
\hline & Temp & -0.23 & 0.15 & -1.52 & 0.136 \\
\hline \multirow[t]{5}{*}{ Glutamine } & Inter & 6.02 & 1.11 & 5.43 & 0.000 \\
\hline & Time & 0.00 & 0.00 & -0.10 & 0.920 \\
\hline & Sex & -0.15 & 0.10 & -1.46 & 0.153 \\
\hline & FL & 0.00 & 0.00 & 2.06 & 0.046 \\
\hline & Temp & 0.00 & 0.05 & -0.82 & 0.419 \\
\hline
\end{tabular}

Table 3 (continued)

\begin{tabular}{|lcrrrc|}
\hline \multirow{2}{*}{ Response } & Parameter & Est & SE & $t$ & $\mathrm{p}$ \\
\hline Glycine & Inter & 6.44 & 2.00 & 3.23 & 0.002 \\
& Time & 0.01 & 0.01 & 1.27 & 0.213 \\
& Sex & -0.14 & 0.18 & -0.78 & 0.440 \\
& FL & -0.01 & 0.00 & -2.75 & $\mathbf{0 . 0 0 9}$ \\
Proline & Temp & -0.02 & 0.10 & -0.16 & 0.871 \\
& Inter & 5.56 & 1.92 & 2.89 & 0.006 \\
& Time & 0.00 & 0.01 & -0.42 & 0.676 \\
& Sex & -0.06 & 0.18 & -0.34 & 0.738 \\
Serine & FL & 0.00 & 0.00 & 0.40 & 0.692 \\
& Temp & -0.05 & 0.09 & -0.48 & 0.631 \\
& Inter & 5.88 & 1.29 & 4.55 & 0.000 \\
Tyrosine & Time & 0.00 & 0.00 & -1.13 & 0.265 \\
& Sex & 0.03 & 0.12 & 0.28 & 0.783 \\
& FL & 0.00 & 0.00 & 2.34 & $\mathbf{0 . 0 2 4}$ \\
& Temp & -0.04 & 0.06 & -0.59 & 0.561 \\
& Inter & 3.02 & 0.85 & 3.55 & 0.001 \\
& Time & 0.00 & 0.00 & -0.44 & 0.667 \\
& Sex & 0.04 & 0.08 & 0.47 & 0.644 \\
& FL & 0.00 & 0.00 & -1.01 & 0.321 \\
& Temp & 0.10 & 0.04 & 2.37 & $\mathbf{0 . 0 2 3}$ \\
\hline
\end{tabular}

Table 4. Fatty acid (FA) profiles (percent contribution, for those that have means above $0.5 \%$ of total FA) from white sharks caught on SMART drumlines in the present study. ND: not detectable; FALD: fatty aldehyde; SFA: saturated FA;

MUFA: monounsaturated FA; PUFA: polyunsaturated FA

\begin{tabular}{|c|c|c|}
\hline Fatty acid & Mean $\pm \mathrm{SE}$ & Range \\
\hline $14: 0$ & $1.87 \pm 0.16$ & $0.81-4.79$ \\
\hline i15:0 & $1.29 \pm 0.09$ & ND-2.54 \\
\hline $16: 1 \omega 9$ & $0.51 \pm 0.11$ & ND-4.51 \\
\hline $16: 1 \omega 7$ & $4.21 \pm 0.44$ & $1.77-10.87$ \\
\hline $16: 0$ & $14.85 \pm 0.38$ & $12.37-24.07$ \\
\hline 16:0FALD & $0.75 \pm 0.03$ & $0.34-1.11$ \\
\hline $17: 1 \omega 8+a 17: 0$ & $0.71 \pm 0.03$ & $0.45-1.46$ \\
\hline $17: 0$ & $0.65 \pm 0.04$ & ND-1.00 \\
\hline $18: 4 \omega 3$ & $0.47 \pm 0.09$ & ND-2.23 \\
\hline $18: 2 \omega 6$ & $0.80 \pm 0.13$ & ND-3.69 \\
\hline $18: 3 \omega 3+C 20$ & $0.46 \pm 0.17$ & ND-6.35 \\
\hline $18: 1 \omega 9$ & $12.14 \pm 0.98$ & $1.63-26.87$ \\
\hline $18: 1 \omega 7$ & $3.94 \pm 0.20$ & $2.29-7.23$ \\
\hline $18: 0$ & $7.99 \pm 0.35$ & $0.39-12.61$ \\
\hline $20: 4 \omega 6$ & $9.35 \pm 0.53$ & $2.11-15.47$ \\
\hline $20: 5 \omega 3$ & $6.19 \pm 0.31$ & $0.10-9.94$ \\
\hline $20: 1 \omega 9$ & $1.08 \pm 0.08$ & ND-2.33 \\
\hline $22: 5 \omega 6$ & $0.94 \pm 0.07$ & ND-1.91 \\
\hline $22: 6 \omega 3$ & $17.47 \pm 0.91$ & $2.46-27.22$ \\
\hline $22: 4 \omega 6$ & $1.61 \pm 0.23$ & ND-9.08 \\
\hline $22: 5 \omega 3$ & $3.50 \pm 0.17$ & ND-5.47 \\
\hline $22: 1 \omega 9$ & $1.04 \pm 0.63$ & $0.11-24.08$ \\
\hline $22: 0$ & $0.52 \pm 0.03$ & ND- 0.75 \\
\hline $24: 1 \omega 9$ & $1.47 \pm 0.07$ & $0.06-2.79$ \\
\hline $24: 0$ & $0.60 \pm 0.04$ & ND-1.14 \\
\hline \multicolumn{3}{|c|}{ Fatty acid groups } \\
\hline$\sum$ SFAs & $27.18 \pm 0.64$ & $17.91-41.45$ \\
\hline $\bar{\Sigma}$ MUFAs & $25.12 \pm 1.48$ & $14.85-46.79$ \\
\hline$\sum$ PUFAs & $40.79 \pm 1.45$ & $12.71-54.20$ \\
\hline
\end{tabular}




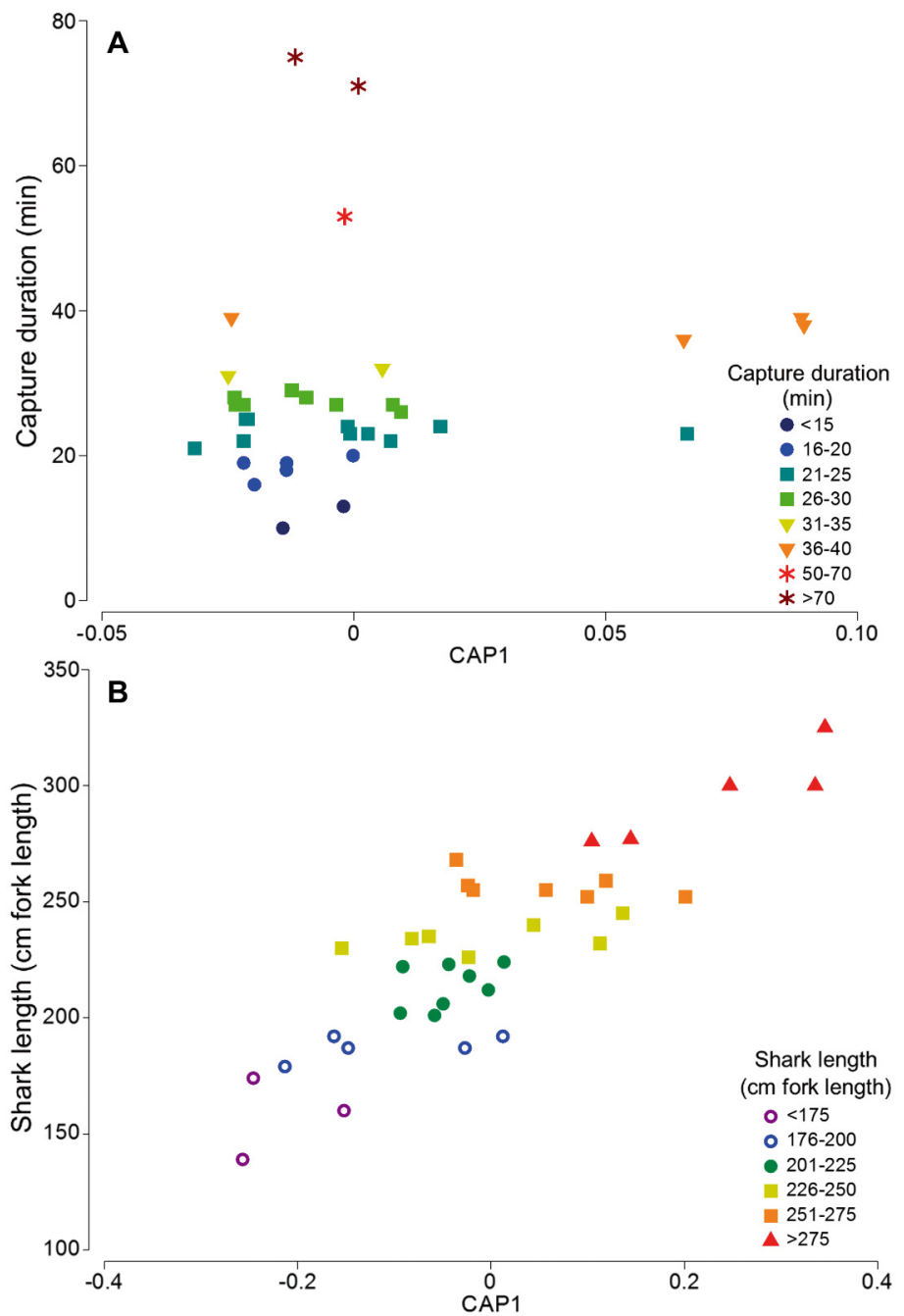

Fig. 2. Canonical analysis of principal coordinates (CAP) of white shark blood plasma fatty acid profiles against (A) capture duration and (B) shark length classed as covariates

as glucose and lactate were affected (Lambert et al. 2018). Our white sharks did not show ketone body sensitivity to capture, suggesting ketone bodies may not be a major source of metabolic fuel under acute stressors. The overall lack of capture duration effect on the indicators in the present study suggests that white sharks do not have an elevated physiological response to short-term capture or that these parameters may not be useful biomarkers for acute capture stress. However, a few non-essential amino acids were affected by shark size, suggesting a relationship between ontogeny and amino acid requirements which may be driven by other growth factors not evaluated here. Alanine, for example, is produced de novo by fish and provides a buffer of skeletal muscle growth in migratory marine fishes (Snyder et al. 2008). Serine, which plays a role in gluconeogenesis
Table 5. Generalized linear model results testing the effects of biological and operational variables on important fatty acids from white sharks caught on SMART drumlines in the present study. SFA: saturated fatty acid; MUFA: monounsaturated fatty acid; PUFA: polyunsaturated fatty acid. Significant p-values in bold. Est: estimate; Inter: intercept; Time: hook time; FL: fork length; Temp: temperature

\begin{tabular}{|c|c|c|c|c|c|}
\hline Response & Parameter & Est & $\mathrm{SE}$ & $t$ & $\mathrm{p}$ \\
\hline \multirow[t]{5}{*}{ 16:0 } & Inter & 2.99 & 0.44 & 6.85 & 0.000 \\
\hline & Time & 0.00 & 0.00 & 0.27 & 0.787 \\
\hline & Sex & 0.03 & 0.04 & 0.61 & 0.549 \\
\hline & FL & 0.00 & 0.00 & -1.23 & 0.229 \\
\hline & Temp & -0.01 & 0.02 & -0.41 & 0.683 \\
\hline \multirow[t]{5}{*}{ 18:0 } & Inter & -0.36 & 0.84 & -0.43 & 0.671 \\
\hline & Time & 0.00 & 0.00 & -0.20 & 0.844 \\
\hline & Sex & 0.40 & 0.09 & 4.67 & 0.000 \\
\hline & $\mathrm{FL}$ & 0.00 & 0.00 & -3.54 & 0.001 \\
\hline & Temp & 0.16 & 0.04 & 3.77 & 0.001 \\
\hline \multirow[t]{5}{*}{$16: 1 \omega 7$} & Inter & 4.25 & 1.40 & 3.04 & 0.005 \\
\hline & Time & 0.00 & 0.01 & -0.49 & 0.631 \\
\hline & Sex & -0.58 & 0.14 & -4.04 & 0.000 \\
\hline & FL & 0.01 & 0.00 & 3.92 & 0.000 \\
\hline & Temp & -0.21 & 0.07 & -3.04 & 0.005 \\
\hline \multirow[t]{5}{*}{$18: 1 \omega 9$} & Inter & 4.09 & 1.19 & 3.44 & 0.002 \\
\hline & Time & 0.00 & 0.00 & 0.04 & 0.970 \\
\hline & Sex & -0.16 & 0.12 & -1.29 & 0.206 \\
\hline & FL & 0.01 & 0.00 & 4.64 & 0.000 \\
\hline & Temp & -0.16 & 0.06 & -2.73 & 0.011 \\
\hline \multirow[t]{5}{*}{$20: 4 \omega 6$} & Inter & 1.55 & 1.15 & 1.35 & 0.187 \\
\hline & Time & 0.00 & 0.00 & 0.10 & 0.923 \\
\hline & Sex & -0.01 & 0.00 & -5.07 & 0.000 \\
\hline & FL & -0.01 & 0.00 & -5.07 & 0.000 \\
\hline & Temp & 0.11 & 0.06 & 1.98 & 0.057 \\
\hline \multirow[t]{5}{*}{$20: 5 \omega 3$} & Inter & 0.77 & 1.08 & 0.71 & 0.483 \\
\hline & Time & 0.00 & 0.00 & -0.26 & 0.796 \\
\hline & Sex & -0.01 & 0.11 & -0.13 & 0.896 \\
\hline & FL & 0.00 & 0.00 & 0.05 & 0.964 \\
\hline & Temp & 0.06 & 0.05 & 1.04 & 0.307 \\
\hline \multirow[t]{5}{*}{$22: 6 \omega 3$} & Inter & 2.42 & 0.98 & 2.47 & 0.019 \\
\hline & Time & 0.00 & 0.00 & 0.83 & 0.413 \\
\hline & Sex & 0.13 & 0.01 & 1.34 & 0.190 \\
\hline & FL & 0.00 & 0.00 & -2.95 & 0.006 \\
\hline & Temp & 0.06 & 0.05 & 1.18 & 0.247 \\
\hline \multirow[t]{5}{*}{ SFA } & Inter & 2.91 & 0.38 & 7.69 & 0.000 \\
\hline & Time & 0.00 & 0.00 & 0.15 & 0.882 \\
\hline & Sex & 0.11 & 0.04 & 2.76 & 0.10 \\
\hline & FL & 0.00 & 0.00 & -2.32 & 0.028 \\
\hline & Temp & 0.03 & 0.02 & 1.56 & 0.129 \\
\hline \multirow[t]{5}{*}{ MUFA } & Inter & 4.27 & 0.86 & 4.96 & 0.000 \\
\hline & Time & 0.00 & 0.00 & -0.07 & 0.942 \\
\hline & Sex & -0.14 & 0.09 & -1.54 & 0.133 \\
\hline & FL & 0.01 & 0.00 & 4.70 & 0.000 \\
\hline & Temp & -0.11 & 0.04 & -2.59 & 0.015 \\
\hline \multirow[t]{5}{*}{ PUFA } & Inter & 3.31 & 0.61 & 5.39 & 0.000 \\
\hline & Time & 0.00 & 0.00 & 0.35 & 0.725 \\
\hline & Sex & 0.07 & 0.06 & 1.05 & 0.310 \\
\hline & FL & 0.00 & 0.00 & -3.31 & 0.002 \\
\hline & Temp & 0.05 & 0.03 & 1.56 & 0.128 \\
\hline
\end{tabular}

(a result of a mobilization of energy from the stress response in fish), is also related to liver function and metabolism; this organ increasingly constitutes a 
large portion of a sharks' biomass as they mature (Hussey et al. 2009, Lucifora et al. 2009). White sharks will also rely more on utilizing stored energy as they age, which was supported by our finding that ketone bodies increased with shark length (Fig. S1). The large biomass and low mass-specific metabolic rates of white sharks support this hypothesis (Carlson et al. 2004), such that they may have limited requirements for increasing the bioavailability of non-essential amino acids for energy production. Since all of the white sharks sampled in the present study were below the estimated size at maturity for this species (Bigelow \& Schroeder 1948, Bruce 1992), it is unlikely that reproductive activity or status played a role in amino acid or FA levels. While all individuals in the present study were immature, the captured sharks were of a relatively large size range (139-325 cm FL) and included young-of-the-year, small juveniles, and large juveniles.

Numerous studies support the conclusion that shark stress-responses to capture can be highly species-specific (Mandelman \& Skomal 2009, Dapp et al. 2016a, Jerome et al. 2018). Although we used a different and novel set of comparative biomarkers for assessing capture stress in sharks than those most commonly used (e.g. blood gas tensions, glucose, and lactate; Marshall et al. 2012), it appears that our results support the notion that shark species can be relatively robust to capture (i.e. tiger sharks; Afonso \& Hazin 2014, Gallagher et al. 2014a,b). Lamnid sharks exhibit physiological and metabolic adaptations for heightened aerobic swimming performance (Bernal et al. 2003a,b). This greater aerobic scope could in turn enhance other aerobic physiological processes such as oxygen delivery and the processing of metabolic end-products of anaerobic metabolism (Wood et al. 2007). Yet within this group of sharks, certain species have been shown to exhibit high stress responses (e.g. mako sharks; Wells \& Davie 1985) but high survivorship to capture (French et al. 2015), which suggests that there is some degree of within-family variation in the response or that there are context-dependent effects (e.g. we used short hooking durations). Considering the high trophic positions occupied by most lamind sharks, as well as the importance of individual white sharks to overall population health (Cooke et al. 2016, Ward et al. 2016), there are important conservation implications when sharks are captured and released. Although these results are preliminary and additional physiological endpoints should be evaluated, our main result may be an encouraging conclusion for white sharks.
We recognize that the current set of biomarkers used (amino acids, FAs) are novel in their application to studies of shark stress (sensu Van Rijn \& Reina 2010, Guida et al. 2016a) and that they may not be as sensitive to capture stress as in other species such as teleosts. A complimentary study recently assessed the physiology of this same sample of white sharks, using more traditional blood biochemistry analytes and found that most parameters did not significantly change with hook time (Tate et al. 2019). Their conclusions align with those from our study using amino acids and FAs; however, we are not able to comment on their true utility as stress indicators on their own, due to the limited sampling window of our study. Thus, amino acids and FAs should be tested under more extreme capture events and used against/correlated with more traditional biomarkers to determine their relative utility. Additionally, the lack of change in biomarker profiles attributed to capture, regardless of whether it is due to their overall suitability as stress biomarkers or the proposed limited stress response, encourages the use of amino acids and FAs in white shark ecology. Both amino acids and FAs were influenced by abiotic (temperature) and biotic (size) factors, mirroring their more traditional use in chondrichthyan ecology (Munroe et al. 2018, Meyer et al. 2019). As such, research teams can confidently apply these biomarkers to questions of white shark diet and habitat use, without the need to incorporate variations in short capture duration, or when precise capture duration may be unknown.

This study represents the first evaluation of white shark response to capture using amino acids and FAs as biomarkers, and one of the first evaluations of white shark physiology in response to capture. Clearly, more work is required to obtain a more comprehensive understanding of this species' physiology and how it changes when exposed to additional fishery settings as well as for more physiological endpoints. This study was limited by the operational aspects of our fishery-independent capture methods (SMART drumlines), which provided short capture durations (averaging $\sim 30 \mathrm{~min}$; ranging 13-75 $\mathrm{min}$ ) and an ability for white sharks to maintain swimming performance while hooked. The ability to swim while hooked will likely reduce or minimize the magnitude of an individual's physiological response to capture (e.g. Guida et al. 2016b). Nevertheless, hook times below this mean can elicit pronounced stress responses in large sharks, and have been linked to effects ranging from physiological change to mortality, using the same general capture technique (Gallagher et al. 2014a). Moreover, the number of indi- 
vidual sharks sampled here greatly exceeds those for most species in other studies. This research suggests that white sharks are likely tolerant of the process of catch and release from SMART drumlines at short to moderate hook times. This result is useful for researchers, managers, and agencies tasked with ensuring high survival rates for this protected species when they are caught and released under research (tagging programs) or management scenarios (bather safety programs), or when they are captured incidentally in non-target fisheries.

Acknowledgements. Project funding and primary support was provided through the Shark Management Strategy by the New South Wales Department of Primary Industries (NSW DPI), Australia. NSW DPI provided scientific (Ref. P01/0059(A), Marine Parks (Ref. P16/0145-1.1), and Animal Care and Ethics (ACEC Ref. 07/08) permits. This project would not have been possible without the dedicated support of Craig Brand and Christopher Gallen and the NSW DPI shark research team. We thank the 3 anonymous reviewers for their comments on the manuscript.

\section{LITERATURE CITED}

Afonso AS, Hazin FH (2014) Post-release survival and behavior and exposure to fisheries in juvenile tiger sharks, Galeocerdo cuvier, from the South Atlantic. J Exp Mar Biol Ecol 454:55-62

* Anderson M, ter Braak CJF (2003) Permutation tests for multi-factorial analysis of variance. J Stat Comput Simul 73:85-113

Aragão C, Corte-Real J, Costas B, Dinis MT, Conceição LEC (2008) Stress response and changes in amino acid requirements in Senegalese sole (Solea senegalensis Kaup 1858). Amino Acids 34:143-148

Ballantyne JS (1997) Jaws: the inside story. The metabolism of elasmobranch fishes. Comp Biochem Physiol B 118: 703-742

Barkley AN, Cooke SJ, Fisk AT, Hedges K, Hussey NE (2017) Capture-induced stress in deep-water Arctic fish species. Polar Biol 40:213-220

Barton BA, Morgan JD, Vijayan MM (2002) Physiological and condition-related indicators of environmental stress in fish. In: Adams SM (ed) Biological indicators of aquatic ecosystem stress. American Fisheries Society, Bethesda, MD, p 111-148

Bell MV, Henderson RJ, Sargent JR (1986) The role of polyunsaturated fatty acids in fish. Comp Biochem Physiol B 83:711-719

Bernal D, Sepulveda C, Mathieu-Costello O, Graham JB (2003a) Comparative studies of high performance swimming in sharks I. Red muscle morphometrics, vascularization and ultrastructure. J Exp Biol 206:2831-2843

* Bernal D, Smith D, Lopez G, Weitz D, Grimminger TADK, Dickson K, Graham JB (2003b) Comparative studies of high performance swimming in sharks II. Metabolic biochemistry of locomotor and myocardial muscle in endothermic and ectothermic sharks. J Exp Biol 206: 2845-2857
Bigelow HB, Schroeder WC (1948) Sharks. In: Tee-Van J, Breder CM, Hildebrand SF, Parr AE, Schroeder WC (eds) Fishes of the western North Atlantic, Part 1. Lancelets, cyclostomes, sharks. Sears Foundation for Marine Research, Yale University, New Haven, CT, p 59-546

B Bligh EG, Dyer WJ (1959) A rapid method of total lipid extraction and purification. Can J Biochem Physiol 37 : 911-917

* Broadhurst MK, Cullis BR (2019). Mitigating the discard mortality of non-target, threatened elasmobranchs in bather-protection gillnets. Fish Res 222:105435

* Brooks EJ, Mandelman JW, Sloman KA, Liss S and others (2012) The physiological response of the Caribbean reef shark (Carcharhinus perezi) to longline capture. Comp Biochem Physiol A Mol Integr Physiol 162:94-100

Bruce BD (1992) Preliminary observations on the biology of the white shark, Carcharodon carcharias, in south Australian waters. Mar Freshw Res 43:1-11

Butcher PA, Peddemors VM, Mandelman JW, McGrath SP, Cullis BR (2015) At-vessel mortality and blood biochemical status of elasmobranchs caught in an Australian commercial longline fishery. Glob Ecol Conserv 3:878-889

Calow P, Forbes VE (1998) How do physiological responses to stress translate into ecological and evolutionary processes? Comp Biochem Physiol A Mol Integr Physiol 120: $11-16$

Carlson JK, Goldman KJ, Lowe CG (2004) Metabolism, energetic demand, and endothermy. In: Carrier JC, Musick JA, Heithaus MR (eds) Biology of sharks and their relatives. CRC Press, Boca Raton, FL, p 203-224

Clarke KR, Gorley RN (2015) Getting started with PRIMER v7. PRIMER-E, Plymouth

Cliff G, Dudley SF (1992) Protection against shark attack in South Africa, 1952-90. Mar Freshw Res 43:263-272

* Cliff G, Dudley SFJ, Davis B (1989) Sharks caught in the protective gill nets off Natal, South Africa. 2. The great white shark Carcharodon carcharias (Linnaeus). S Afr J Mar Sci 8:131-144

* Cooke SJ, Hogan ZS, Butcher PA, Stokesbury MJ and others (2016) Angling for endangered fish: Conservation problem or conservation action? Fish Fish 17:249-265

* Costas B, Conceição LE, Aragão C, Martos JA, Ruiz-Jarabo I, Mancera JM, Afonso A (2011) Physiological responses of Senegalese sole (Solea senegalensis Kaup, 1858) after stress challenge: effects on non-specific immune parameters, plasma free amino acids and energy metabolism. Aquaculture 316:68-76

C Curtis TH, McCandless CT, Carlson JK, Skomal GB, Kohler NE, Natanson LJ, Pratt HL Jr (2014) Seasonal distribution and historic trends in abundance of white sharks, Carcharodon carcharias, in the western North Atlantic Ocean. PLOS ONE 9:e99240

Danylchuk AJ, Suski CD, Mandelman JW, Murchie KJ, Haak CR, Brooks AM, Cooke SJ (2014) Hooking injury, physiological status and short-term mortality of juvenile lemon sharks (Negaprion bevirostris) following catch-and-release recreational angling. Conserv Physiol 2:cot036

* Dapp DR, Huveneers C, Walker TI, Drew M, Reina RD (2016a) Moving from measuring to predicting bycatch mortality: predicting the capture condition of a longlinecaught pelagic species. Front Mar Sci 2:126

* Dapp DR, Walker TI, Huveneers C, Reina RD (2016b) Respiratory mode and gear type are important determinants of elasmobranch immediate and post-release mortality. Fish Fish 17:507-524 
Dapp DR, Huveneers C, Walker TI, Mandelman J, Kerstetter D, Reina RD (2017) Using logbook data to determine the immediate mortality of blue sharks (Prionace glauca) and tiger sharks (Galeocerdo cuvier) caught in the commercial US pelagic longline fishery. Fish Bull 115:27-41

Dicken ML, Booth AJ (2013) Surveys of white sharks (Carcharodon carcharias) off bathing beaches in Algoa Bay, South Africa. Mar Freshw Res 64:530-539

₹ Ducatez S (2019) Which sharks attract research? Analyses of the distribution of research effort in sharks reveal significant non-random knowledge biases. Rev Fish Biol Fish 29:355-367

Dulvy N K, Fowler S L, Musick JA, Cavanagh RD, Kyne PM, Harrison LR, Pollock CM (2014) Extinction risk and conservation of the world's sharks and rays. eLife 3:e00590

French RP, Lyle J, Tracey S, Currie S, Semmens JM (2015) High survivorship after catch-and-release fishing suggests physiological resilience in the endothermic shortfin mako shark (Isurus oxyrinchus). Conserv Physiol 3:cov044

Gallagher AJ, Huveneers CP (2018) Emerging challenges to shark-diving tourism. Mar Policy 96:9-12

Gallagher AJ, Kyne PM, Hammerschlag N (2012) Ecological risk assessment and its application to elasmobranch conservation and management. J Fish Biol 80:1727-1748

Gallagher AJ, Serafy JE, Cooke SJ, Hammerschlag N (2014a) Physiological stress response, reflex impairment, and survival of five sympatric shark species following experimental capture and release. Mar Ecol Prog Ser 496:207-218

Gallagher AJ, Orbesen ES, Hammerschlag N, Serafy JE (2014b) Vulnerability of oceanic sharks as pelagic longline bycatch. Global Ecol Conserv 1:50-59

Gallagher AJ, Staaterman ER, Cooke SJ, Hammerschlag N (2017a) Behavioural responses to fisheries capture among sharks caught using experimental fishery gear. Can J Fish Aquat Sci 74:1-7

Gallagher AJ, Skubel RA, Pethybridge HR, Hammerschlag N (2017b) Energy metabolism in mobile, wild-sampled sharks inferred by plasma lipids Conserv Physiol 5: cox002

* Gladyshev MI, Sushchik NN, Tolomeev AP, Dgebuadze YY (2018) Meta-analysis of factors associated with omega-3 fatty acid contents of wild fish. Rev Fish Biol Fish 28: $277-299$

Guida L, Walker TI, Reina RD (2016a) The adenylate energy charge as a new and useful indicator of capture stress in chondrichthyans. J Comp Physiol B 186:193-204

Guida L, Walker TI, Reina RD (2016b) Temperature insensitivity and behavioural reduction of the physiological stress response to longline capture by the gummy shark, Mustelus antarcticus. PLOS ONE 11:e0148829

Gulak SJB, de Ron Santiago AJ, Carlson JK (2015) Hooking mortality of scalloped hammerhead Sphyrna lewini and great hammerhead Sphyrna mokarran sharks caught on bottom longlines. Afr J Mar Sci 37:267-273

Guyomard D, Perry C, Tournoux PU, Cliff G, Peddemors V, Jaquemet $S$ (2019) An innovative fishing gear to enhance the release of non-target species in coastal shark-control programs: The SMART (shark management alert in realtime) drumline. Fish Res 216:6-17

Hammerschlag N, Gallagher AJ, Lazarre DM (2011) A review of shark satellite tagging studies. J Exp Mar Biol Ecol 398:1-8

Heard M, Van Rijn JA, Reina RD, Huveneers C (2014) Impacts of crowding, trawl duration and air exposure on the physiology of stingarees (family: Urolophidae) Conserv Physiol 2:cou040

* Hsieh SL, Chen YN, Kuo CM (2003) Physiological responses, desaturase activity, and fatty acid composition in milkfish (Chanos chanos) under cold acclimation. Aquaculture 220:903-918

KHussey NE, Cocks DT, Dudley SFJ, McCarthy ID, Wintner SP (2009) The condition conundrum: application of multiple condition indices to the dusky shark Carcharhinus obscurus. Mar Ecol Prog Ser 380:199-212

Huveneers C, Meekan MG, Apps K, Ferreira LC, Pannell D, Vianna GM (2017) The economic value of shark-diving tourism in Australia. Rev Fish Biol Fisheries 27:665-680

* Huveneers C, Apps K, Becceri-Garcia E, Bruce B and others (2018) Future research directions on the 'elusive' white shark. Front Mar Sci 5:455

* Jerome JM, Gallagher AJ, Cooke SJ, Hammerschlag N (2018) Integrating reflexes with physiological measures to evaluate coastal shark stress response to capture. ICES J Mar Sci 75:796-804

Jorgensen SJ, Reeb CA, Chapple TK, Anderson S and others (2010) Philopatry and migration of Pacific white sharks. Proc R Soc B 277:679-688

* Kock A, O'Riain MJ, Mauff K, Meÿer M, Kotze D, Griffiths C (2013) Residency, habitat use and sexual segregation of white sharks, Carcharodon carcharias in False Bay, South Africa. PLOS ONE 8:e55048

Lambert FN, Treberg JR, Anderson WG, Brandt C, Evans AN (2018) The physiological stress response of the Atlantic stingray (Hypanus sabinus) to aerial exposure. Comp Biochem Physiol Part A Mol Integr Physiol 219:38-43

* Li P, Mai K, Trushenski J, Wu G (2009) New developments in fish amino acid nutrition: towards functional and environmentally oriented aquafeeds. Amino Acids 37:43-53

* Li XM, Yuan JM, Fu SJ, Zhang YG (2016) The effect of sustained swimming exercise on the growth performance, muscle cellularity and flesh quality of juvenile qingbo (Spinibarbus sinensis). Aquaculture 465:287-295

Lowe CG, Blasius ME, Jarvis ET, Mason TJ, Goodmanlowe GD, O'Sullivan JB (2012) Historic fishery interactions with white sharks in the Southern California Bight. In: Domeier ML (ed) Global perspectives on the biology and life history of the white shark. CRC Press, Boca Raton, FL, p 169-186

*Lucifora LO, García VB, Menni RC, Escalante AH, Hozbor NM (2009) Effects of body size, age and maturity stage on diet in a large shark: ecological and applied implications. Ecol Res 24:109-118

* Lyons K, Jarvis ET, Jorgensen SJ, Weng K, O'Sullivan J, Winkler C, Lowe CG (2013) The degree and result of gillnet fishery interactions with juvenile white sharks in southern California assessed by fishery-independent and -dependent methods. Fish Res 147:370-380

* Ma XY, Qiang J, He J, Gabriel NN, Xu P (2015) Changes in the physiological parameters, fatty acid metabolism, and SCD activity and expression in juvenile GIFT tilapia (Oreochromis niloticus) reared at three different temperatures. Fish Physiol Biochem 41:937-950

Malcolm H, Bruce BD, Stevens JD (2001) A review of the biology and status of white sharks in Australian waters. Report to Environment Australia, Marine Species Protection Program. CSIRO Marine Research, Hobart

*Mandelman JW, Skomal GB (2009) Differential sensitivity to capture stress assessed by blood acid-base status in five carcharhinid sharks. J Comp Physiol B 179:267 
Marshall H, Field L, Afiadata A, Sepulveda C, Skomal G, Bernal D (2012) Hematological indicators of stress in longline-captured sharks. Comp Biochem Physiol A Mol Integr Physiol 162:121-129

Mazeaud MM, Mazeaud F, Donaldson EM (1977) Primary and secondary effects of stress in fish: some new data with a general review. Trans Am Fish Soc 106:201-212

Metcalf VJ, Gemmell NJ (2005) Fatty acid transport in cartilaginous fish: absence of albumin and possible utilization of lipoproteins. Fish Physiol Biochem 31:55-64

Meyer L, Pethybridge H, Nichols PD, Beckmann C, Bruce BD, Werry JM, Huveneers C (2017) Assessing the functional limitations of lipids and fatty acids for diet determination: the importance of tissue type, quantity, and quality. Front Mar Sci 4:369

Meyer L, Pethybridge H, Nichols PD, Beckmann C, Huveneers C (2019) Abiotic and biotic drivers of fatty acid tracers in ecology: a global analysis of chondrichthyan profiles. Funct Ecol 33:1243-1255

Milligan CL (1997) The role of cortisol in amino acid mobilization and metabolism following exhaustive exercise in rainbow trout (Oncorhynchus mykiss Walbaum). Fish Physiol Biochem 16:119-128

Moyes CD, Fragoso N, Musyl MK, Brill RW (2006) Predicting postrelease survival in large pelagic fish. Trans Am Fish Soc 135:1389-1397

Munroe S, Meyer L, Heithaus M (2018) Dietary biomarkers in shark foraging and movement ecology. In: Carrier J, Heithaus M, Simpfendorfer C (eds) Shark research: emerging technologies and applications for the field and laboratory. CRC Press, Boca Raton, FL, p 1-24

Nazimi L, Robbins WD, Schilds A, Huveneers C (2018) Comparison of industry-based data to monitor white shark cage-dive tourism. Tour Manage 66:263-273

Osako K, Saito H, Hossain MA, Kuwahara K, Okamoto A (2006) Docosahexaenoic acid levels in the lipids of spotted mackerel Scomber australasicus. Lipids 41:713-720

Prohaska BK, Bethea DM, Poulakis GR, Scharer RM, Knotek R, Carlson JK, Grubbs RD (2018) Physiological stress in the smalltooth sawfish: effects of ontogeny, capture method, and habitat quality. Endang Species Res 36: 121-135

RStudio Team (2016) RStudio: integrated development for R. RStudio Inc., Boston, MA. www.rstudio.com (accessed July 2017)

Reid DD, Robbins WD, Peddemors VM (2011) Decadal trends in shark catches and effort from the New South Wales, Australia, Shark Meshing Program 1950-2010. Mar Fresh Res 62:676-693

Editorial responsibility: Brendan Godley, University of Exeter, Cornwall Campus, UK
Skomal GB, Braun CD, Chisholm JH, Thorrold SR (2017) Movements of the white shark Carcharodon carcharias in the North Atlantic Ocean. Mar Ecol Prog Ser 580:1-16

Snyder GS, Gaylord TG, Barrows FT, Hardy RW (2008) Carnosine supplementation on an all-plant protein diet for rainbow trout Oncorhynchus mykiss. In: Aquaculture America 2008, book of Abstracts. World Aquaculture Society, Sorrento, LA, p 369

* Talwar B, Brooks EJ, Mandelman JW, Grubbs RD (2017) Stress, post-release mortality, and recovery of commonly discarded deep-sea sharks caught on longlines. Mar Ecol Prog Ser 582:147-161

Tate RD, Cullis BR, Smith SDA, Kelaher BP and others (2019) The acute physiological status of white sharks (Carcharodon carcharias) exhibits minimal variation after capture on SMART drumlines. Conserv Physiol 13:coz042

* Tocher DR (2003) Metabolism and functions of lipids and fatty acids in teleost fish. Rev Fish Sci 11:107-184

*Van Rijn JA, Reina RD (2010) Distribution of leukocytes as indicators of stress in the Australian swellshark, Cephaloscyllium laticeps. Fish Shellfish Immunol 29: 534-538

Vijayan MM, Pereira C, Grau EG, Iwama GK (1997) Metabolic responses associated with confinement stress in tilapia: the role of cortisol. Comp Biochem Physiol C Pharmacol Toxicol Endocrinol 116:89-95

Ward TD, Algera DA, Gallagher AJ, Hawkins E and others (2016) Understanding the individual to implement the ecosystem approach to fisheries management. Conserv Physiol 4:cow005

Wells RM, Davie PS (1985) Oxygen binding by the blood and hematological effects of capture stress in two big gamefish: mako shark and striped marlin. Comp Biochem Phyiol 81:643-646

*Wendelaar Bonga SE (1997) The stress response in fish. Physiol Rev 77:591-625

Whitney NM, White CF, Anderson PA, Hueter RE, Skomal GB (2017) The physiological stress response, postrelease behavior, and mortality of blacktip sharks (Carcharhinus limbatus) caught on circle and J-hooks in the Florida recreational fishery. Fish Bull 115:532-544

*Wood CM, Kajimura M, Bucking C, Walsh PJ (2007) Osmoregulation, ionoregulation and acid-base regulation by the gastrointestinal tract after feeding in the elasmobranch (Squalus acanthias). J Exp Biol 210:1335-1349

* Wood CM, Walsh PJ, Kajimura M, McClelland GB, Chew SF (2010) The influence of feeding and fasting on plasma metabolites in the dogfish shark (Squalus acanthias). Comp Biochem Physiol A Mol Integr Physiol 155:435-444

Submitted: June 20, 2019; Accepted: September 30, 2019 Proofs received from author(s): December 2, 2019 